The outcome of an attachment-based infant mental health therapeutic play programme on infant temperament, parent-infant relationship \& maternal reflective functioning

Joy Malinit*

Philippine Children's Medical Center, University of the East Ramon Magsaysay Memorial Medical Center

${ }^{*}$ Corresponding author.

doi: 10.1192/bjo.2021.713

Aims. In the Philippines, there is a need for preventive, early intervention programs for perinatal and infant mental health. This is the first local study that investigated an attachment-based, therapeutic play programme (Baby Bonding) on infant temperament, parent-infant relationship and maternal reflecting functioning.

Background. This study was an effort towards bridging the "10/90 gap in infant mental health research" wherein $90 \%$ of the world's infants are born in low- middle-income countries (Population Reference Bureau, $2013 \mathrm{~b}$ ) and "only $10 \%$ of the worldwide spending on health research is directed towards the problems that primarily affect the poorest $90 \%$ of the world's population (Tomlinson et al., 2014).

Method. Phase I involved local validation of the Parent-Rated Outcome Measures (PROM)- Infant Characteristics Questionnaire (ICQ), Mother Object Relations Scale (MORS) and Parental Reflective Functioning Questionnaire (PRFQ).Healthy motherbaby dyads, from the low socio-economic stratum, were screened using Parent Evaluation of Developmental Status (PEDS) and Hospital and Anxiety Depression Scale (HADS).

Phase II carried out randomized controlled design wherein mother-baby dyads were enrolled either in the usual care group or the 6-weekly Baby Bonding intervention.

Result. 102 mothers answered the PROM. Their responses constituted the training set of the study tools. Baseline responses of the mothers from the usual care $(\mathrm{N}=51)$ and intervention $(\mathrm{N}=53)$ groups formed the evaluation set for the Filipino- translated instruments. In both the training and evaluation sets, if certain identified questions were to be removed from the PROM, better and acceptable Cronbach values were consistently generated.

There were no statistical differences on parent-infant relationship and reflective functioning between the usual care and intervention group. There was movement of the intervention group from high-challenging onto intermediate to low-levels of challenging temperament in the dull-dimension of the ICQ after 6 sessions. In comparison, infants in the control group remained in the high-challenging temperament after 6 weeks of usual care.

Conclusion. Linguistically validated study instruments (ICQ and MORS) provided accurate assessments of infant temperament and parent-child relationship. The Filipino-translated PRFQ has limited validity in evaluating parental reflective functioning (RF). "On-line" measures that video mother-baby interactions could have better captured changes in RF. As measured by the dull dimension of the ICQ, the Baby Bonding programme improved sociability of the infants ( 7 months or younger).

\section{Maternal immune activation generates anxiety in offspring: A translational meta-analysis}

Ursula Matos $^{1 \star}$, Laiana Azevedo Quagliato ${ }^{1}$ and Antonio Egidio Nardi ${ }^{2}$

${ }^{1}$ Institute of Psychiatry, Federal University of Rio de Janeiro and ${ }^{2}$ Full Professor of Psychiatry, Federal University of Rio de Janeiro ${ }^{\star}$ Corresponding author.

doi: 10.1192/bjo.2021.714
Aims. Maternal immune activation (MIA) during pregnancy is recognized as an etiological risk factor for various psychiatric disorders, such as schizophrenia, major depressive disorder, and autism. Prenatal immune challenge may serve as a "disease primer" into an altered trajectory of fetal brain development that, in combination with other genetic and environmental factors, may ultimately result in the emergence of different psychiatric conditions. However, the association between MIA and the offspring's chances of developing anxiety disorders is less clear. To examine the effect of MIA on offspring anxiety, a systematic review and meta-analysis of the preclinical literature was conducted.

Method. A systematic search of the PubMed, Web of Science, PsycINFO, and Cochrane Library electronic databases was performed using the PRISMA and WHO methodologies for systematic reviews. Studies that investigated if MIA during rodent's pregnancy could cause anxiety symptoms in offspring were included.

Result. Overall, the meta-analysis showed that MIA induced anxiety behavior in offspring. The studies provide strong evidence that prenatal immune activation impacts specific molecular targets, synapse formation and function, and a disbalance in neurotransmission that could be related to the generation of offspring anxiety. Future research should further explore the role of MIA in anxiety endophenotypes.

Conclusion. According to this meta-analysis, MIA plays an important role in the pathophysiological mechanisms of anxiety disorders and provides a promising therapeutic target.

\section{Exploring the mental health impact on private flat} owners in residential buildings with external combustible cladding

\section{William Martin*}

Warrington \& Halton NHS Trust, UK Cladding Action Group ${ }^{\star}$ Corresponding author.

\section{doi: 10.1192/bjo.2021.715}

Aims. To explore the mental health of private flat owners in residential buildings with external combustible cladding, which require remediation following the 2017 Grenfell tower fire.

The speed at which the fire at Grenfell spread, killing 72 people, is largely attributed to external combustible cladding. It is increasingly suspected that there are ongoing profound effects on the mental health of people living in flats with external combustible cladding like Grenfell both in the UK and abroad. The issue has left flat owners facing severe financial hardship, the threat of bankruptcy and concerns about safety in their own homes.

Method. An exploratory 'Google Forms' online mental health survey comprising multiple choice and free text questions over 47 sections was distributed to flat owners in affected buildings. The survey remained open for 6 weeks to allow response. 550 individual responses were studied.

Result. 550 individuals completed the survey, from 143 buildings across 45 UK councils.

As a direct result of external combustible cladding:

89.5\% said their mental health had deteriorated,

$22.5 \%$ reported having suicidal feelings or a desire to self-harm, $71.1 \%$ reported having difficulty sleeping,

$93.8 \%$ said they were suffering from worry and anxiety,

$59.6 \%$ used coping strategies to deal with their situation,

$35.1 \%$ said that existing physical and mental health conditions

had been exacerbated,

$84.1 \%$ said they cannot move on with their lives and 
$57.9 \%$ of people had concerns about seeking help or treatment for mental/physical health problems caused by their situation during the pandemic.

In addition, free text responses reflected feelings of anxiety and low mood attributed to the constant fear of fire, and an inability to plan families and future homes. One person said, "I have been left utterly broken by this. My mental and physical health has worsened, I have severe anxiety, depression and PTSD. I struggle each day to keep myself alive."

Conclusion. Safe housing is a basic human right. The results show the current situation is having a detrimental impact on flat owners' mental health and makes a strong case for the provision of specific services offering support - particularly given it is 3.5 years since Grenfell and a viable solution for all is yet to be found.

Perspectives on implementing HIIT interventions for service users in inpatient mental health settings: a qualitative study investigating patient, carer and staff attitudes

\section{Rebecca Martland ${ }^{1 *}$, Juliana Onwumere ${ }^{2}$, Fiona Gaughran ${ }^{3}$} and Brendon Stubbs ${ }^{4}$

${ }^{1}$ Institute of Psychiatry, Psychology and Neuroscience (IoPPN), Department of Psychosis Studies; ${ }^{2}$ Institute of Psychiatry, Psychology and Neuroscience (IoPPN), Department of Psychology,National

Psychosis Unit, South London and Maudsley NHS Foundation Trust; ${ }^{3}$ Institute of Psychiatry, Psychology and Neuroscience (IoPPN), Department of Psychosis Studies, National Psychosis Unit, South London and Maudsley NHS Foundation Trust and ${ }^{4}$ Institute of Psychiatry, Psychology and Neuroscience (IoPPN), Department of Psychological Medicine, South London and Maudsley NHS

Foundation Trust

${ }^{*}$ Corresponding author.

doi: 10.1192/bjo.2021.716

Aims. High intensity interval training (HIT) may improve a range of physical and mental health outcomes among people with severe mental illnesses (SMI). However, there is limited data on patients' reported attitudes towards HIIT and its implementation within inpatient settings, and there remains an absence of data on attitudes towards HIIT from informal family carers of service users and healthcare professionals, who both have key roles to play in facilitating recovery outcomes in service users. This study sought to qualitatively investigate, in inpatients with SMI, carer and staff groups, perspectives on implementing HIIT interventions for patient groups in inpatient settings.

Method. Seven focus groups and one individual interview were conducted. These included three focus groups held with inpatients with SMI $(n=12)$, two held with informal carers $(n=15)$, and two held with healthcare professionals working in inpatient settings (n =11). An additional individual interview was conducted with one patient participant. The focus group schedule comprised openended questions designed to generate discussion and elicit opinions surrounding the introduction of HIIT on inpatient mental health wards. Data were subject to a thematic analysis.

Result. Two key themes emerged from the data, across all participants, that reflected the 'Positivity' in the application of HIIT interventions in psychiatric inpatient settings with beliefs that it would help patients feel more relaxed, build their fitness, and provide a break from the monotony of ward environment. Moreover, the short length of HIIT sessions was deemed appealing to mitigate against difficulties that many inpatients can experience with motivation, interest and attention, and was considered to be more appealing than more lengthy forms of exercise, which may require greater physical exertion. The second theme related to 'Implementation concerns', that reflected subthemes about i) low patient motivation, particularly with older participants, those administered many medications, and for those with less positive memories of exercise ii) patient safety, including concerns surrounding the intensity of HIIT and inclusion of patients with physical health comorbidities and iii) practical logistical factors, including having access to the right sports clothing and staff availability to supervise HIIT.

Conclusion. HIIT for inpatients with SMI was actively endorsed by patients, carers and healthcare professionals. Patient safety and baseline motivation levels, and practical service considerations were all noted as potential barriers to successful implementation and are worth considering in preparation for trialing a new intervention.

\section{Patient experience of telemedicine in addictions}

Soraya Mayet ${ }^{1 \star}$, Iain Mccaw ${ }^{2}$, Zeeshan Hashmani ${ }^{3}$, Zuzana Drozdova ${ }^{1}$, Amelia Gledhill ${ }^{1}$, Samreen Arshad ${ }^{4}$, Shumaila Shahbaz ${ }^{1}$ and Thomas Phillips ${ }^{5}$

${ }^{1}$ Humber Teaching NHS FT; ${ }^{2}$ South West Yorkshire Partnership NHS Foundation Trust; ${ }^{3}$ Tees, Esk and Wear Valleys NHS

Foundation Trust; ${ }^{4}$ Rotherham Doncaster and South Humber NHS Foundation Trust and ${ }^{5}$ University of Hull

${ }^{\star}$ Corresponding author.

doi: 10.1192/bjo.2021.717

Aims. Opioid dependence has high risks and opioid substitution treatment (OST) improves outcomes and reduces deaths. Attendance at addiction specialist prescribers may be limited, particularly in rural areas. Telemedicine, such as videoconferencing, can reduce travel and improve access and attendance. Pre-COVID-19, we started a telemedicine service for patients with opioid dependence, prescribed opioid substitution treatment, requiring addiction specialist prescriber consultations. We present patient experience and assess whether patients recommend telemedicine.

Method. Health Research Authority approval for Randomized Controlled Trial of Telemedicine versus Face-to-Face (control) appointments in large semi-rural community addictions service $(2500 \mathrm{~km} 2)$ using a modified Hub-and-Spoke (outreach). Adult opioid dependent patients prescribed OST and attending outreach clinics recruited. Participants received two consultations in group. Telemedicine delivered using Skype-for-business videoconferencing. Patients attended outreach clinic, where an outreach worker undertook drug testing and telemedicine conducted via the outreach workers laptop. Specialist addiction prescribers located remotely, at the Hub. Patients self-completed NHS Friends and Family Test (FFT) immediately after appointment, separate from the wider research study. Data collected Sept 2019- March 2020 (pre-COVID-19 lockdown), Microsoft Excel analysis, with qualitative thematic free-text analysis.

Result. Thirty completed FFTs were received, of which all participants were 'extremely likely' $(\mathrm{n}=19 ; 67 \%)$ or 'likely' $(\mathrm{n}=11 ; 37 \%)$ to recommend the Telemedicine service to friends or family, if they needed similar care. Two themes for reasons for recommending the service were; 1 . Convenience (reduced travel, reduced travel time and reduced travel costs) and 2. Supportive Staff (including listening, caring and good support). One patient mentioned it is a convenient way to communicate with medical staff, saving time and effort'. Regarding Telemedicine appointments, most participants responded that the timing of telemedicine 